0271-5198(95)00027-5

\title{
ANNOUNCEMENT
}

\section{THE VIENNA PHYSICAL MEDICINE AWARD 1995}

Eligibility : $\quad$ All academic scientists working in Physical Medicine or in related fields

Conditions : $\quad$ Regulations of the Vienna Physical Medicine Award

Deadline : $\quad 1^{\text {st }}$ August 1995

Prize : $\quad$ Yearly scientific award of 10.000 DM (6000 US \$)

plus travel subsidy of maximum $1000 \mathrm{DM}$

Sponsor : $\quad$ Zimmer Elektromedizin GmbH, Neu-Ulm, Germany

Institution : $\quad$ Chair of Physical Medicine and Rehabilitation,

University of Vienna, Austria

Submission : $\quad$ Unpublished original scientific papers

from all aspects of Physical Medicine, in English (maximal length: 20 typed pages).

Papers are automatically submitted for publication in the "European Journal of Physical Medicine and Rehabilitation"

All manuscripts must be anonymous and include:

a) name and address of author(s)

b) short C.V.(s)

c) statement that participant is author

d) statement confirming that the participant is entitled to publish the manuscript and agreeing to its publication.

Ceremony : $\quad$ in Vienna, date to be announced

Correspondence : Doz. Dr. V. Fialka

Dept. Phys. Med. Rehab.

Währinger Gürtel 18-20

AKH, 1090 Vienna, Austria 